\title{
Automatic Modulation Classification Based on In-Phase Quadrature Diagram Constellation Combined with a Deep Learning Model
}

\author{
Jean Baptiste Bi Gouho ${ }^{1, *}$, Sidibé Karim¹, Boko Aka² and Michel Babri ${ }^{3}$ \\ ${ }^{1}$ Laboratoire de Mathématiques Informatique, Université Nangui Abrogoua, Abidjan, \\ Côte d'Ivoire \\ ${ }^{2}$ Institut de Recherche sur les Energies Nouvelles, Université Nangui Abrogoua, \\ Abidjan, Côte d'Ivoire \\ 3Institut National Polytechnique Félix Houphouët Boigny, Yamoussoukro, Côte \\ d'Ivoire
}

\begin{abstract}
Article Type: Article
Article Citation: Jean Baptiste Bi Gouho, Sidibé Karim, Boko Aka, Michel Babri. Automatic modulation classification based on In phase quadrature diagram constellation combined with a deep learning model. Indian Journal of Science and Technology. 2020; 13(02), 200-212. D0l: 10.17485/ijst/2020/ v013i02/148648
\end{abstract}

Received date: November 4, 2019

Accepted date: December 13, 2019

*Author for correspondence: Jean Baptiste Bi Gouho gouhobi@ yahoo.fr@gouhobi@yahoo.fr

\begin{abstract}
Objectives: This study aims to present a framework for Automatic Modulation recognition using Deep learning without feature extraction. Methods: We study seven modulations using the In-Phase Quadrature constellation polluted by Additive White Gaussian Noise. We apply the K-means algorithm to normalize data transmitted and polluted by noise; the new diagram obtained is considered as an image and coded in pixel before entering in a Deep Neural Network where we apply $20 \%$ dropout on hidden layers to avoid overfitting. The simulation is carried out in Matlab. Findings: Experiment performed on selected modulations following the proposed framework gives a good percentage of recognition equal to $96.12 \%$. Our algorithm Deep Neural Network imaGe gives the best performance results at epoch equal to 2,000,000. Applications: The outcome will be beneficial for researchers in Software-Defined Radio for civilian and military applications like electronic attacks and electronic protection.
\end{abstract}

Keywords: Modulations, I-Q Diagram Constellation, Clustering, Deep Neural Network, Dropout.

\section{Introduction}

Modulation recognition has many commercial applications in areas such as space communications and cellular telecommunications where it is used as a Software-Defined Radio. Software-Defined Radios use a blind modulation recognition system to reduce system overhead and increase system accuracy. 
The advantage of using machine learning in Automatic Modulation Classification is that system that uses a decision tree for this purpose with several detection levels is not an easy thing [1]. In [2] used a Neural Network to improve the percentage of modulation recognition during classification. In [3] used fuzzy c-means combined with Neural Networks to classify different types of modulations, but the proposed technique required features extractions before entering in the Neural Network. In [4] proposed a classification method using the combination of two clustering methods, namely the k-means and the $\mathrm{k}$-center for the modulation classification, one used to initialize the data and the other one come up in support for decision-making using as a basis of the I-Q constellation diagram.

In [5] used the K-nearest neighbor method which is a learning method that uses two phases, the learning phase and the classification phase [6] for the classification of modulations. In most of the methods proposed in many papers [3-7], authors combine two methods to solve the classification problem. In [8] proposes a new model that combines extraction and classification as a single entity with a deep learning model.

In this study, we propose an approach based on the use of the Gaussian noise polluted $\mathrm{I}-\mathrm{Q}$ diagram as basic information for use in modulation recognition problem-solving. We propose to use the k-means algorithm to normalize the noise-polluted diagram and then enter the new modified diagram into a hidden multi-layered Neural Network and apply an adequate dropout on our Neural Network to prevent overfitting.

\section{Literature Survey}

In this section, we will present the algorithms and models known in the literature.

\section{1. k-means Algorithm}

The principle of $\mathrm{k}$-means is to minimize the distortions that are call cost.

$$
\cos t=\min _{C}\left[\sum_{k=1}^{K} \sum_{x_{i} \in c_{k}}\left\|x_{i}-\mu_{K}\right\|^{2}\right]
$$

where $\mathrm{x}_{\mathrm{i}}$ is a number of clusters $\mathrm{C}_{\mathrm{k}}$ and $\mu_{\mathrm{k}}$ is the centroid of each cluster $\mathrm{C}_{\mathrm{k}}$.

$$
\mu_{K}=\frac{1}{N_{K}} \sum_{x_{i} \in K} x_{i}
$$

The fundamental purpose of the clustering method is to assign observations into a cluster.

\subsection{Neural Network}

Neural Networks are derived from biology and are today one of the most explored areas of artificial intelligence due to its applications in several fields [9]. Figure 1 shows us a typical Neural Network. 


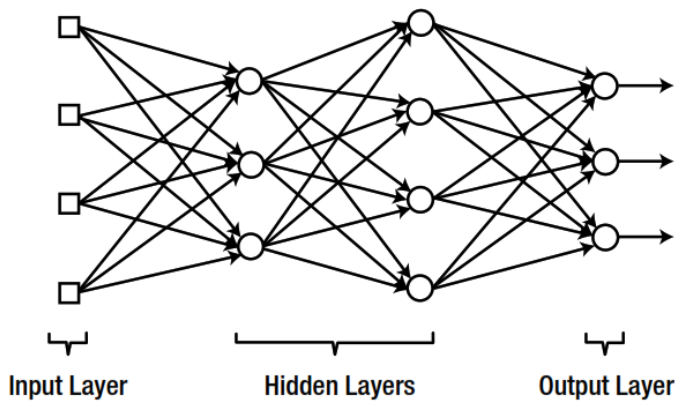

FIGURE 1. Neural network.
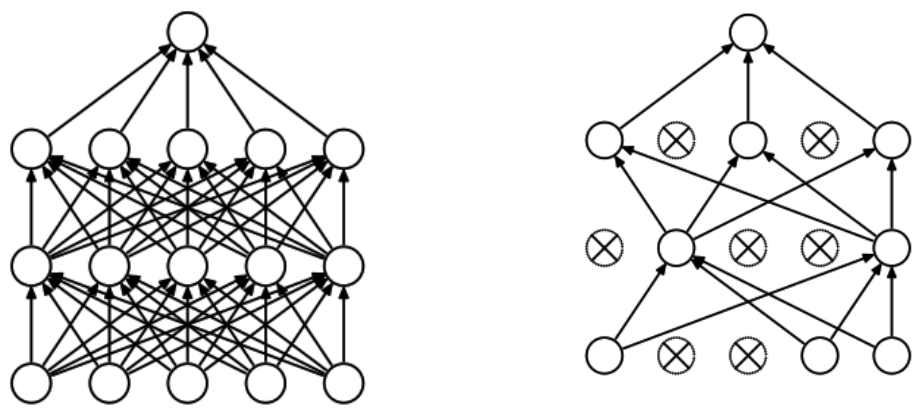

FIGURE 2. (a) Standard neural network. (b) After applying dropout.

In the application of Neural Networks in problem-solving, one of the major problems is overfitting. To overcome the problem of overfitting, we use dropout techniques [10]. This has been applied by [11] to Recurrent Neural Networks (RNNs) to ward off the problem of random selection of certain nodes and the deactivation of unselected nodes.

The principle is illustrated in Figure 2 (a) and (b).

\subsection{Model of [3]}

In [3] proposed a model for automatic modulation classification. This is illustrated in Figure 3.

In this model, the modulated signal during transmission is polluted by Gaussian noise which can cause an improbable demodulation. At first, they apply a clustering algorithm that is fuzzy c-means to try to normalize the data polluted by the noise. After extracting

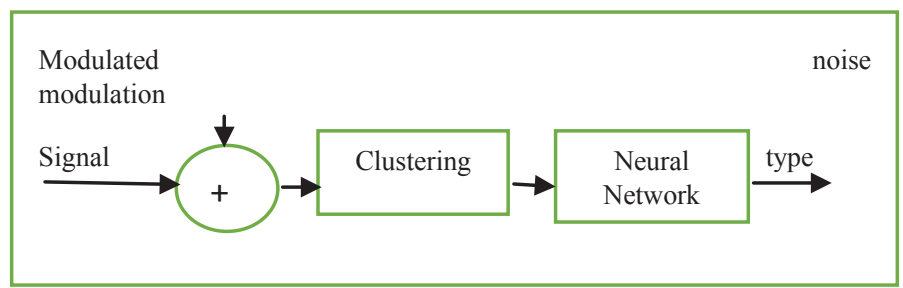

FIGURE 3. Model of [3]. 
some parameters, these parameters are introduced into a Neural Network to recognize the modulation.

\subsection{Classification Model Proposed by [8]}

Traditional models used in the literature $[2-3,7]$ used to extract certain parameters which will then be used to feed the Neural Network. The problem occurs because it was necessary to choose good parameters at the risk of feeding the Neural Network with bad parameters which were crucial because a wrong choice will lead to erroneous results due to the parameters received. In [8] proposed a model that combines extraction and classification phases into a single block to classify modulations used in unmanned aerial vehicles.

This model is shown in Figure 4 (a) and (b).

\section{Proposed Model for Classification}

Our model proposed in this study is a combination of approaches proposed by [3] and that of [8]. This is illustrated in Figure 5.

\subsection{Signal Model}

In this study, used signals are in Intermediate Frequency (IF) and are corrupted by Additive White Gaussian Noise (AWGN). The signals used are the same type as those proposed by [12]. The transmitted signal $r(t)$ is as follows:

$$
r(t)=s(t)+n(t)
$$

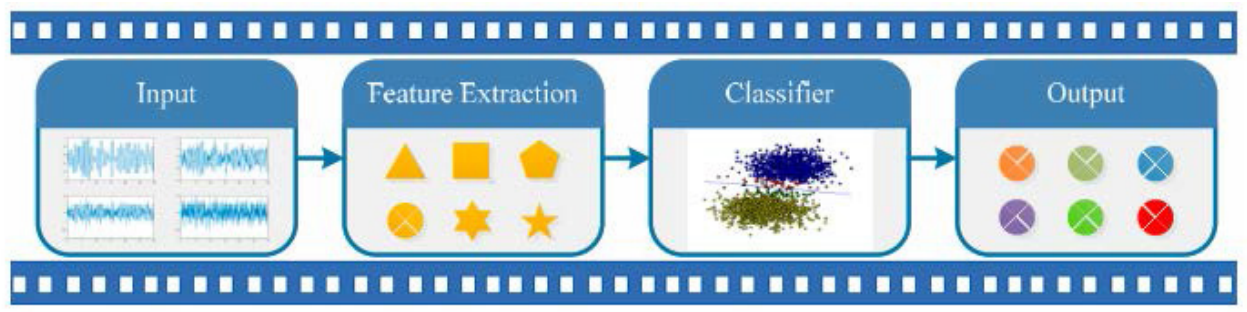

(a)

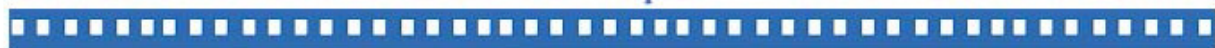

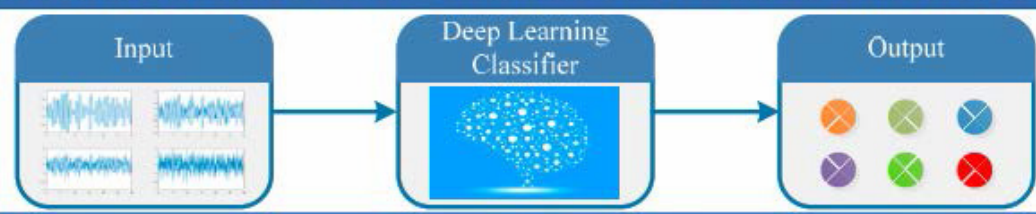

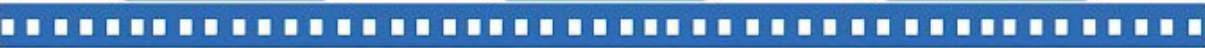

(b)

FIGURE 4. (a) Traditional classification model. (b) Classification model proposed by [8]. 


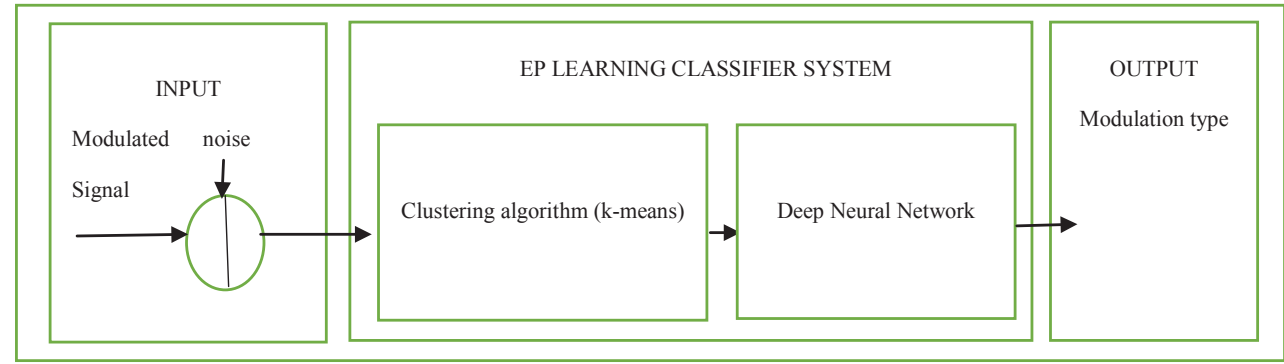

FIGURE 5. Proposed model for classification.

where $s(t)$ is the modulated signal and $n(t)$ is the Gaussian noise affecting that signal.

The modulation set studied in this study includes M-QAM $(M=4,8,16,32)$ and M-PSK $(M=2,8,16)$ signals, $s(t)$ is expressed as

$$
s(t)=A_{m} \sum_{n} a_{n} g\left(t-n T_{s}\right) \cos \left[2 \pi\left(f_{c}+f_{m}\right) t+\varphi_{0}+\varphi_{m}\right]
$$

where $A_{m}, a_{n}, T_{s}, f_{c}, f_{m}, \varphi_{0}, \varphi_{m}$ are the modulation amplitude, symbol sequence, symbol period, carrier frequency at IF, modulation frequency, initial phase, and modulation phase, respectively. $g(t)$ is the gate function

$$
g(t)=\left\{\begin{array}{l}
1 \text { si } 1 \leq t \leq T_{s} \\
0 \text { sinon }
\end{array}\right.
$$

\subsection{Application of the k-means Algorithm}

We know that the purpose of clustering is to assign observations to a cluster. In [13] suggested that the receiver could use the geometry of the constellation of the modulated signal to deduct the modulation. To have this result, they propose to consider the matrix $\mathrm{X}$ where

$$
X=\left[x_{1} x_{2} . . x_{j} \ldots x_{N}\right]^{T}
$$

$\mathrm{N}$ represents a set of points and

$$
X_{j}=\left[x_{j I}+x_{j Q}\right]^{T}
$$

where $x_{j I}$ is the component In Phase and $x_{j Q}$ is the component in Quadrature.

When the modulation used is 16 QAM with $\mathrm{N}=10000$ and $\mathrm{S} / \mathrm{N}=4 \mathrm{~dB}$, Figure 6 shows the representation of 16 QAM modulated signal constellation.

The received signal polluted by the noise gives the following representation in Figure 7.

The application of the k-means algorithm gives us the result of Figure 8. 


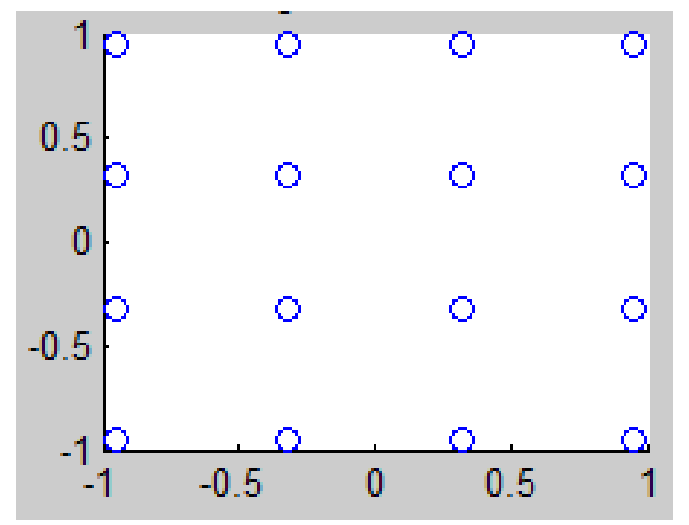

FIGURE 6. Representation of the modulated signal in 16 QAM.

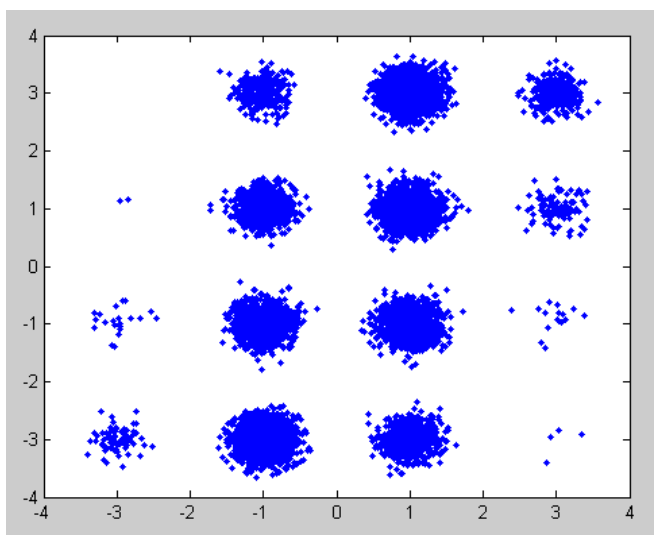

FIGURE 7. Representation of the transmitted signal polluted by noise.

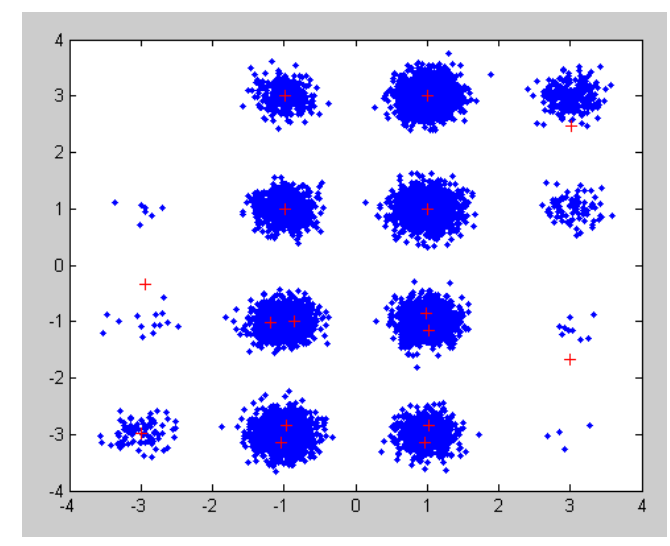

FIGURE 8. Result of the application of the k-means algorithm. 


\subsection{Coding of the Constellation Diagram}

In [3] and [4] have demonstrated that the application of a clustering algorithm (fuzzy c-means, k-means, k-center) to normalize data polluted by Gaussian noise only cannot be used to recognize the modulation used. It will be necessary to couple given results to a Neural Network to fulfill the task of classification $[3,14]$.

Our proposed algorithm is a supervised learning case where all modulations are known by receiver and recognition is done through the use of the I-Q constellation diagram which will be considered as a pixel-coded picture. For example, if the modulation used is 16 QAM, the original constellation will be coded as follows in Figure 9.

Constellation representing the signal polluted by noise and after application of the $\mathrm{k}$-means algorithm will be coded as follows in Figure 10.

The result shows that our constellation diagram is encoded as a $7 x 7$ matrix.

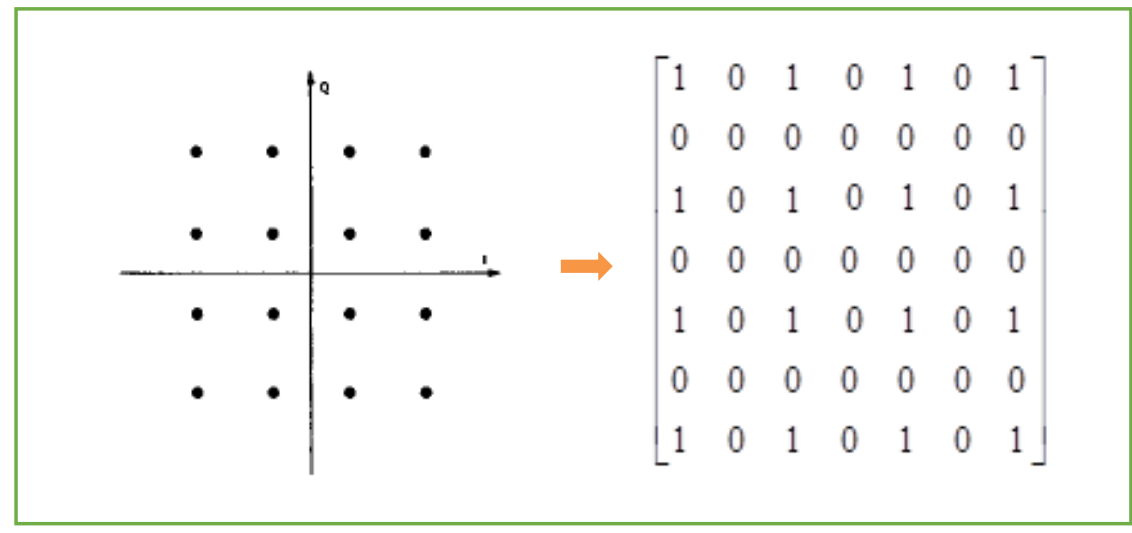

FIGURE 9. 16 QAM constellation coded.

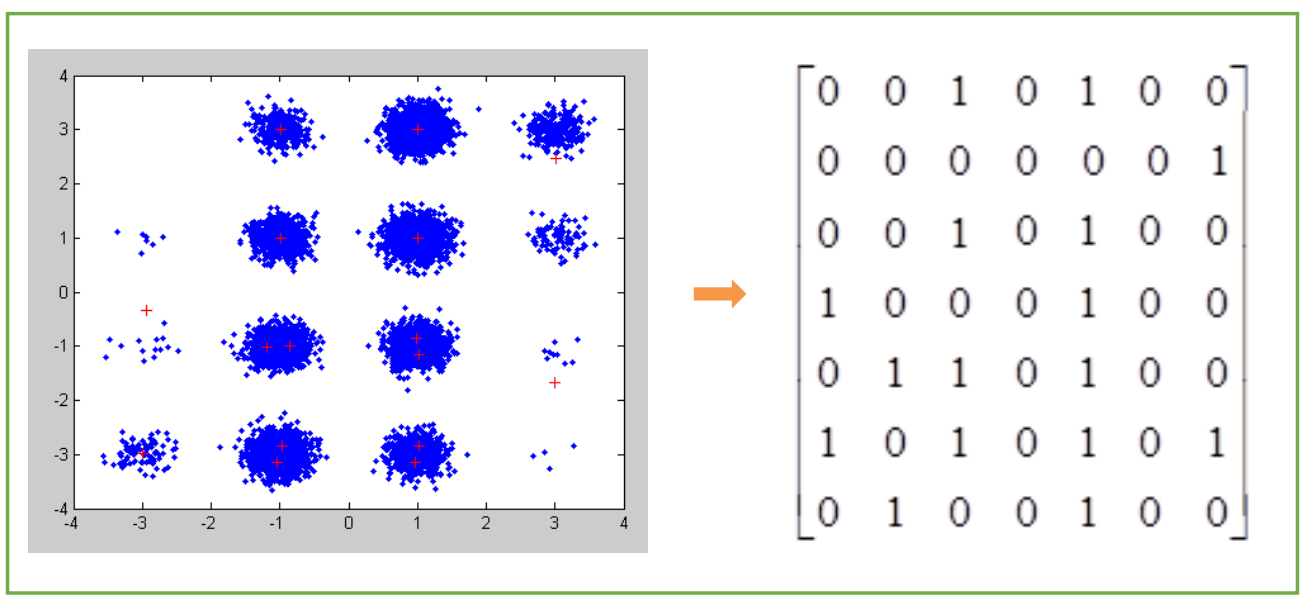

FIGURE 10. Coded representation of modulated signal in 16 QAM after application of k-means. 


\subsection{The Aspect of Our Neural Network}

To solve our problem of classification, we consider a Deep Neural Network with three hidden layers. Each hidden layer consists of 40 nodes. Neural network has 49 nodes of entry and 7 output corresponding to 7 classes corresponding also to our 7 modulations chosen which are the most used in many papers $[3-5,7,13]$. As each modulation constellation can be set on a matrix 7x7, we set 49 input nodes. Our three hidden layers represent the deep model of the Neural Network proposed by [14] which has a single hidden layer. Figure 11 shows the architecture of our neural network.

$\mathrm{W} 1, \mathrm{~W} 2, \mathrm{~W} 3, \mathrm{~W} 4$, respectively, represent the weights of the matrices of the input nodes-hidden 1 layer, hidden 1 layer-hidden2 layer, hidden 2 layer-hidden3 layer, hidden 3 layer-output layer. The activation function for neural network is softmax function.

$$
y_{i}=\varphi\left(v_{i}\right)=\frac{e^{v_{i}}}{\sum_{k=1}^{M} e^{v_{k}}}
$$

\subsection{Proposed Algorithm}

Our algorithm has been named Deep Neural Network imaGe (DNNG) because it uses I-Q constellation diagrams as a pixel-coded image to feed the neural network. A normality test [4] is performed on the signal to know whether we are dealing with a single carrier signal or multiple carrier signal type. In this article, we will deal with the case of single carrier signal.

After applying the k-means clustering algorithm, this information is entered into the Deep Neural Network for modulation recognition.

To avoid overfitting, we drop out $20 \%$ of each hidden node.

Proposed algorithm is presented in Figure 12.

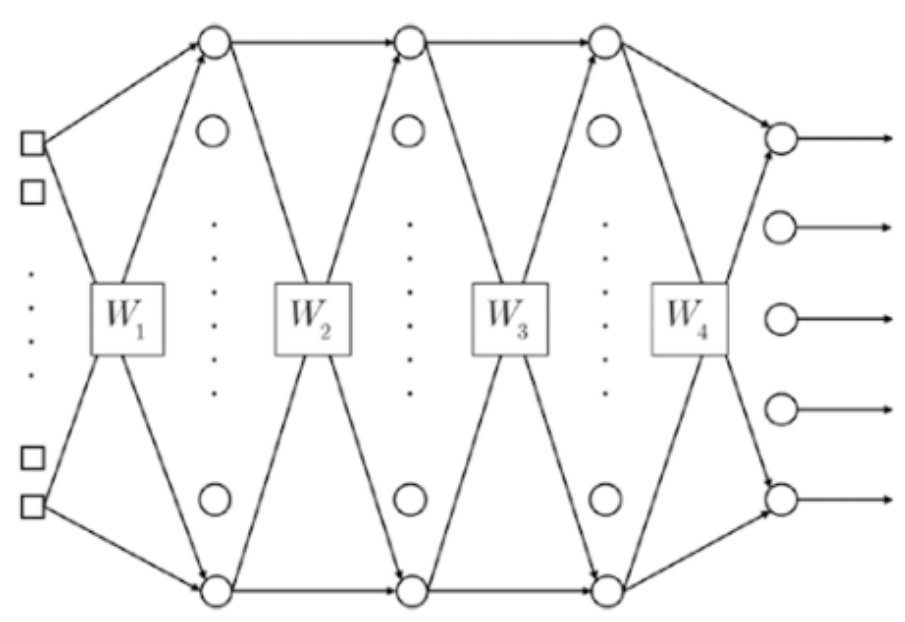

FIGURE 11. Deep Neural Network used for classification. 


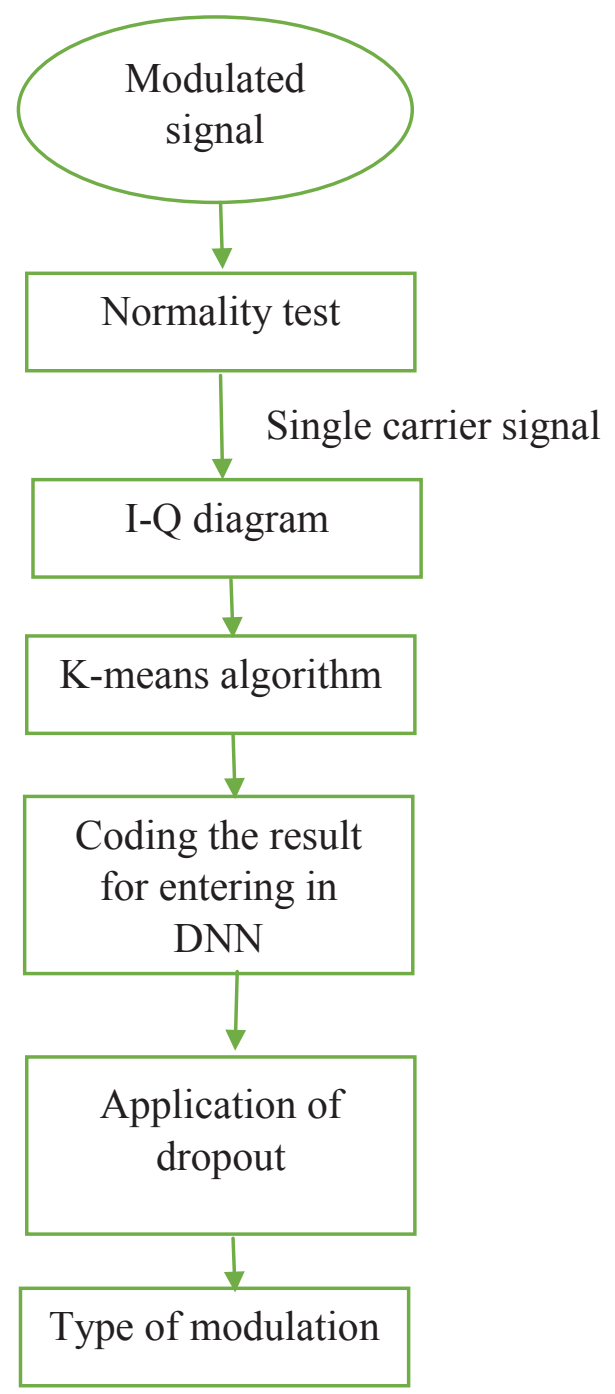

FIGURE 12. Flow chart of proposed algorithm DNNG.

\section{Results and Discussion}

For the learning phase of our Neural Network, we set the learning rate for the proper functioning at $\alpha=0.01(0 \prec \alpha \leq 1)$; a bad choice of this parameter will lead to a slow convergence of the neural network [15].

Our simulation has been done with these parameters:

- PC running Windows 10

- 8 Gb of RAM

- Processor Intel core i5 $7^{\text {th }}$ generation

- Programming language is MATLAB 2013a 
TABLE 1. Order of entrance of modulations in the Deep Neural Network

\begin{tabular}{ll}
\hline Order & Diagram I-Q pollute by noise \\
\hline 1 & 16-QAM \\
2 & 4-QAM \\
3 & 8-PSK \\
4 & 16-PSK \\
5 & BPSK \\
6 & 8-QAM \\
7 & 32-QAM \\
\hline
\end{tabular}

In several papers $[3-5,7,13,16]$ dealing with the automatic classification of modulations, the weak classification results generally occur when there are low values of $S / N$.

That is why we used to test our model at $\mathrm{S} / \mathrm{N}=4 \mathrm{~dB}$.

Table 1 shows the order of entrance of our seven modulations chosen in the Deep Neural Network.

Table 2 shows all the modulation recognition percentages of the Neural Network starting from an epoch value equal to 10,000 .

The curve in Figure 13 shows that our Neural Network converges well, but after the value of epoch equals 2,000,000, it enters in overfitting.

It is at this value that our Neural Network gives the best results, with a general recognition percentage of our seven modulations equaling $96.12 \%$.

\subsection{Comparisons with Other Results}

To compare our results with those which exist in the literature, we will do it with four modulations because it is these modulations that several authors have used $[3-5,14]$. But the comparison with other results of the literature is difficult as was mentioned in papers $[7,13]$ because there are no standard databases of modulations that everyone should use to facilitate comparison and the parameters of experiments differ from one author to another.

Table 3 presents the main results of some methods proposed in the literature.

Table 3 summarizes results obtained and shows an improved performance in terms of modulation recognition of our DNNG method. We have especially compared it to our first

TABLE 2. Results given by the Deep Neural Network

\begin{tabular}{llllll}
\hline Modulations & \multicolumn{5}{c}{ Epoch } \\
\cline { 2 - 6 } & $\mathbf{1 0 , 0 0 0}$ & $\mathbf{1 0 , 0 0 0}$ & $\mathbf{2 , 0 0 0 , 0 0 0}$ & $\mathbf{3 , 0 0 0 , 0 0 0}$ & $\mathbf{4 , 0 0 0 , 0 0 0}$ \\
\hline 16 QAM & 0.9609 & 0.9937 & 1.0000 & 1.0000 & 1.0000 \\
4 QAM & 0.9842 & 0.9995 & 1.0000 & 1.0000 & 1.0000 \\
8 PSK & 0.9739 & 0.9997 & 1.0000 & 1.0000 & 1.0000 \\
16 PSK & 0.6010 & 0.4379 & 0.7300 & 0.5999 & 0.5773 \\
BPSK & 0.9953 & 1.0000 & 1.0000 & 1.0000 & 1.0000 \\
8 QAM & 0.9934 & 1.0000 & 1.0000 & 1.0000 & 1.0000 \\
32 QAM & 0.9976 & 0.9999 & 1.0000 & 1.0000 & 1.0000 \\
\hline
\end{tabular}




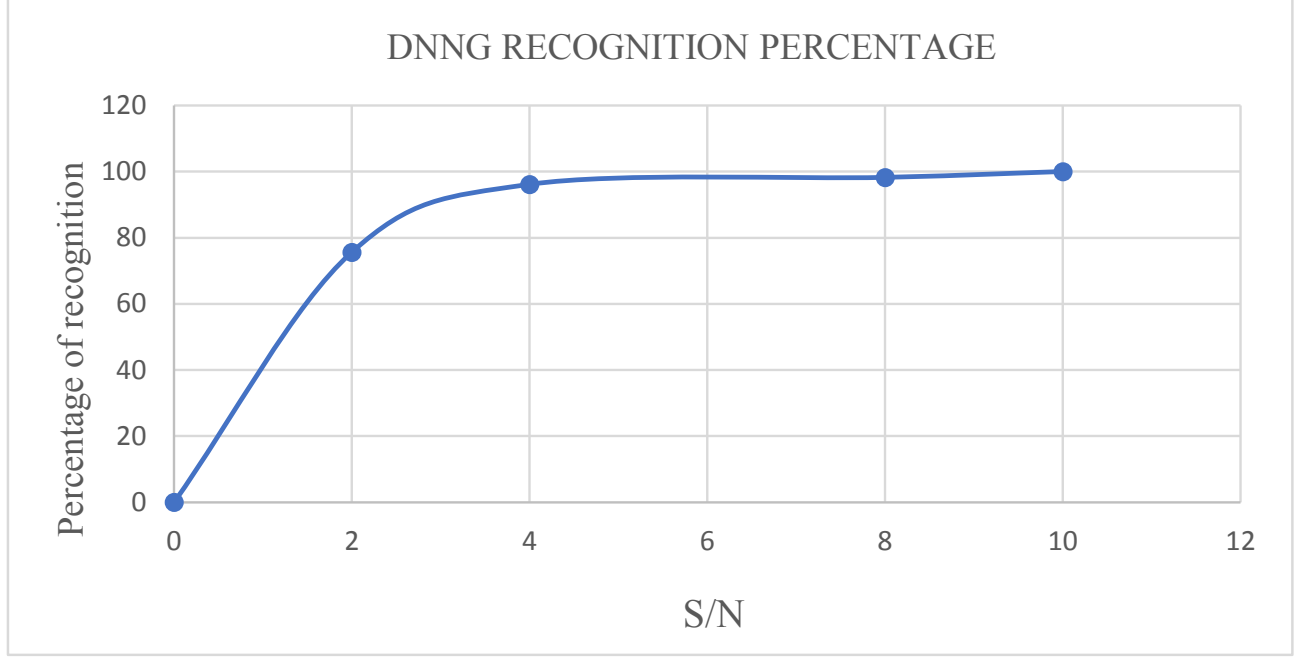

FIGURE 13. Convergence of the Deep Neural Network.

TABLE 3. Comparison with other methods at $\mathrm{S} / \mathrm{N}=4 \mathrm{~dB}$

\begin{tabular}{llllll}
\hline \multirow{2}{*}{ Method } & \multicolumn{5}{c}{ Modulation } \\
\cline { 2 - 6 } & BPSK & 8 PSK & 4 QAM & 32 QAM & Average \\
\hline Fuzzy clustering method (FCM) of [3] & $100 \%$ & $95.8 \%$ & $93.6 \%$ & $16 \%$ & $70.6 \%$ \\
Clustering combined with ANN of [3] & $100 \%$ & $100 \%$ & $100 \%$ & $73.6 \%$ & $91.2 \%$ \\
k-means and k-center of [15] & $100 \%$ & $87 \%$ & $96 \%$ & $95 \%$ & $94.5 \%$ \\
KNN of [5] & $100 \%$ & $82.73 \%$ & $59.99 \%$ & $60 \%$ & $75.68 \%$ \\
ANNG of [14] & $92.91 \%$ & $99.17 \%$ & $96.87 \%$ & $99.98 \%$ & $97.23 \%$ \\
DNNG of [14] & $100 \%$ & $100 \%$ & $100 \%$ & $100 \%$ & $100 \%$ \\
\hline
\end{tabular}

Artificial Neural Network imaGe (ANNG) which used a Neural Network of one hidden layer; this new model DNNG which uses a Neural Network of three hidden layers gives better results.

We have a significant improvement in the level of the iterations because with the ANNG, it was necessary to go to value of epoch equal to 4000000 before having good results, but with the DNNG at 2,000,000, its gives better results. DNNG brings a gain in CPU resource consumption compared to ANNG.

The curve in Figure 14 shows the evolution of the ratio of the percentage of recognition of the seven modulations used in our DNNG model concerning the signal-to-noise ratio $(\mathrm{S} / \mathrm{N})$.

\section{Conclusion}

In this study, we presented a model for Automatic Modulation Classification that we named DNNG which is a model of deep learning from another model that we proposed 


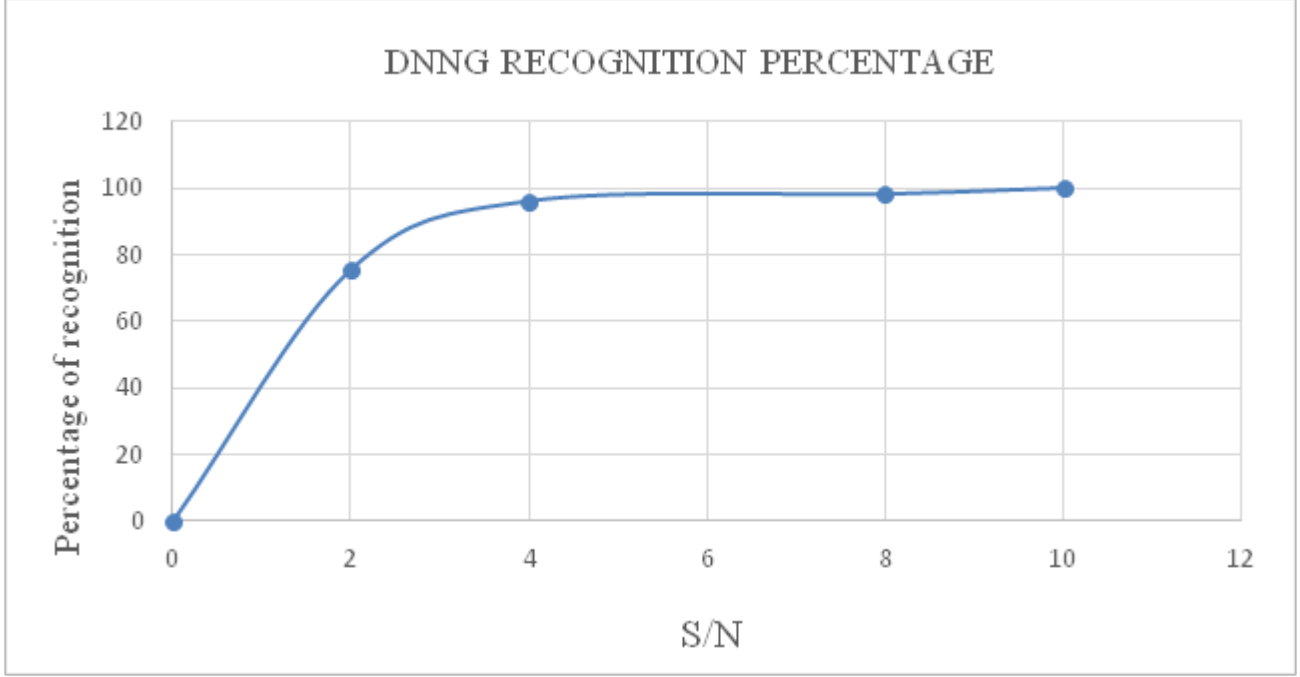

FIGURE 14. Recognition percentage of DNNG on our seven modulations recognition.

ANNG for automatic modulation classification. The new model presented improved the percentage of modulation recognition for low $\mathrm{S} / \mathrm{N}$ values and showed a reduction in the epoch value (divided into 2) used in the learning phase of the neural network compared to the first proposed method. In perspective, we propose to build a Convolutional Neuron Network (CNN) always on our basic model of use of the In-Phase Quadrature diagram constellation considered as reference information.

\section{References}

1. Automatic modulation classification principales, algorithms and applications. https://www. amazon.com/Automatic-Modulation-Classification-Principles-Applications/dp/1118906497. Date accessed: 16/02/2015.

2. Nandi AK, Azzouz EE. Modulation recognition using artificial neural network. Signal Processing. 1997; 56(2), 165-175.

3. Liu A, Zhu Q. Automatic modulation classification based on the combination of clustering and neural network. Journal of China Universities of Posts and Telecommunications. 2011; 18(4), 1-6.

4. I-Q diagram utilization in a novel modulation classification technique for cognitive radio applications. https://jwcn-eurasipjournals.springeropen.com/articles/10.1186/1687-14992013-289. Date accessed: 20/12/2013.

5. Zhu Z, Aslam MW, Nandi AK. Automatic modulation classification using combination of genetic programming and KNN. IEEE Transactions on Wireless Communications. 2012; 11(8), 2742-2750.

6. Too J, Abdullah AR, Saad NM. Classification of hand movements based on discrete wavelet transform and enhanced feature extraction. International Journal of Advanced Computer Science and Applications (IJACSA). 2019; 10(6), 83-89.

7. Wong MLD, Nandi AK. Automatic digital modulation recognition using artificial neural network and genetic algorithm. Signal Processing. 2004; 84(2), 351-365. 
8. Zhang D, Ding W, Zhang B, Xie C, Li H, Liu C, Han J. Automatic modulation classification based on deep learning for unmanned aerial vehicules. MDPI Journal Sensors. 2018; 18, 924.

9. LeCun Y, Bengio Y, Hinton G. Deep learning. Nature. 2015; 521(7553), 436-444.

10. Survey of dropout methods for Deep Neural Networks. https://arxiv.org/abs/1904.13310. Date accessed : 25/10/2019.

11. Srivastava N, Hinton G, Krizhevsky A, Sutskever I, Salakhutdinov R. Dropout: a simple way to prevent neural networks from overfitting. Journal of Machine Learning Research. 2014; 15, 1929-1958.

12. Zhou S, Yin Z, Wu Z, Chen Y, Zhao N, Yang Z. A robust modulation classification method using convolutional neural networks. EURASIP Journal on Advances in Signal Processing. 2019; 21, $1-15$.

13. Mobasseri BG. Digital modulation classification using constellation shape. Signal Processing. 2000; 80, 251-277.

14. Gouho JBB, Mélèdje D, Aka B, Babri M. New approach of automatic modulation classification based on in phase-quadrature diagram combined with artificial neural network. International Journal of Advanced Computer Science and Applications. 2019; 10(7), 141-145.

15. Matlab deep learning: with machine learning neural network and artificial intelligence. https:// www.apress.com/gp/book/9781484228449. Date accessed: 2017.

16. Modulation classification based on extensible neural networks. https://www.hindawi.com/ journals/mpe/2017/6416019/. Date accessed: 2017. 